\title{
Prognostic value of systemic immune-inflammatory index in survival outcome in gastric cancer: a meta-analysis
}

\author{
Shifeng Fu ${ }^{1,2}$, Jin Yan ${ }^{1,2}$, Yuyong Tan ${ }^{1,2}$, Deliang Liu ${ }^{1,2}$ \\ ${ }^{1}$ Department of Gastroenterology, the Second Xiangya Hospital, Central South University, Changsha, China; ${ }^{2}$ Research Center of Digestive Disease, \\ Central South University, Changsha, China \\ Contributions: (I) Conception and design: All authors; (II) Administrative support: D Liu; (III) Provision of study materials or patients: S Fu, J Yan; \\ (IV) Collection and assembly of data: S Fu, J Yan; (V) Data analysis and interpretation: All authors; (VI) Manuscript writing: All authors; (VII) Final \\ approval of manuscript: All authors. \\ Correspondence to: Prof. Deliang Liu, MD, PhD. Department of Gastroenterology, the Second Xiangya Hospital, Central South University, No. 139 \\ Renmin Road, Furong District, Changsha 410011, China; Research Center of Digestive Disease, Central South University, No. 139 Renmin Road, \\ Furong District, Changsha 410011, China. Email: delianglilu@csu.edu.cn.
}

Background: In recent years, many studies have reported that the systemic immune-inflammatory index (SII) can be used to predict the prognosis of cancer patients; however, this finding remains controversial in gastric cancer (GC). Therefore, the purpose of this study was to systematically and comprehensively probe the prognostic role of SII in GC.

Methods: Relevant publications were extracted from PubMed, EMBASE, Cochrane Library databases, and WANFANG DATA (Chinese database). The included studies had patients with pathologically confirmed GC and long-term follow-up data. The patient's outcome was death, recurrence, or status at the end of follow-up. The studies included randomized controlled tests, case-control studies, or cohort studies using a multivariate proportional hazard model adjusted for survival outcomes. Cochran's Q test and Higgins' I-squared statistic were performed to assess heterogeneity. Publication bias was assessed by visual inspection of a Begg's funnel plot.

Results: A total of 6,925 patients in 11 studies were included. The pooled hazard ratio (HR) indicated that a higher SII value was significantly associated with worse overall survival (OS) [HR: 1.53, 95\% confidence interval (CI): 1.27-1.83] and worse disease-free survival (DFS) (HR: 1.57, 95\% CI: 1.24-1.97) in GC patients. In the subgroup analysis, the HR was 1.72 (95\% CI: $1.51-1.95)$ and 1.27 (95\% CI: 0.96-1.67) in the group of patients aged $<59$ and $\geq 59$ years, respectively.

Conclusions: The pooled HR indicates that a higher SII in younger patients with GC predicts a poor prognosis. In elderly patients with GC, the prognostic role of SII needs further research.

Keywords: Stomach neoplasms; systemic immune-inflammatory index (SII); prognosis; inflammation index; survival outcome

Submitted Jul 01, 2020. Accepted for publication Dec 07, 2020.

doi: 10.21037/jgo-20-252

View this article at: http://dx.doi.org/10.21037/jgo-20-252

\section{Introduction}

Gastric cancer (GC) is the most common tumor of the digestive system, and the third leading cause of cancerrelated deaths (1). Despite the advancements in medical technologies and treatments, the prognosis of GC patients remains poor. About $50-90 \%$ of patients die because of GC recurrence after curative resection (2), and the median overall survival (OS) of advanced GC is only 10-12 months (3). The American Joint Committee on Cancer (AJCC)-TNM staging system is widely used by clinicians to evaluate the prognosis of patients with GC. However, patients with the same TNM stage may have different prognoses after 
the same treatment. Therefore, more accurate evaluation indicators are needed. Prognostic evaluation should be performed after the initial diagnosis and resection of GC to determine whether more radical treatments should be adopted to achieve a better clinical outcome.

In recent years, many studies have reported that the inflammation-related hematological indices, such as lymphocyte-to-monocyte ratio (LMR), neutrophil-tolymphocyte ratio (NLR), platelet-to-lymphocyte ratio (PLR), and systemic immune-inflammatory index (SII), can evaluate the prognosis of cancer patients (4-6). Patients with different levels of these indices may have different prognoses, even if they are in the same TNM stage (7). SII, which consist of lymphocytes, neutrophils, and platelets (platelets $\times$ neutrophils/lymphocytes), is believed to be superior to the other indices $(8,9)$. Chen et al. (10) believed that SII is more effective in distinguish patients whose prognoses are different within the same TNM group. Moreover, some meta-analyses indicated that elevated SII levels are related to the poor prognosis of ovarian cancer, breast cancer, colorectal cancer, and urinary system tumors (10-13), and SII exceeding the threshold can also predict worse OS in hepatocellular carcinoma, GC, esophageal squamous cell carcinoma, urinary system tumors, lung cancer, and melanoma (14). Furthermore, Fest et al. (15) conducted an investigation in healthy people $>45$ years old and found that elevated SII is a risk factor for solid tumors, and that people with high baseline SII level have a 30\% higher risk of developing a solid cancer than those with low SII level. These results indicate that patients with high level SII may have tumors that cannot be detected at baseline (15). Although a lot of evidence has indicated that SII has great potential in predicting the prognosis of cancer patients, the findings in GC patients remain controversial. While most studies showed that SII is related to the poor prognosis of GC patients (16-21), some studies show that SII has no predictive effect (22-24). Hence, we conducted a meta-analysis of published articles to further explore the relationship between SII and the prognosis in GC patients. We present the following article in accordance with the Preferred Reporting Items for Systematic Reviews and Meta-Analyses (PRISMA) reporting checklist (25) (available at http://dx.doi.org/10.21037/jgo-20-252).

\section{Methods}

\section{Search strategy}

This meta-analysis has been registered at INPLASY.COM, with DOI number 10.37766/inplasy2020.5.0021. It was carried out in accordance with the PRISMA guidelines (25). Because the concept of SII was only proposed in 2014, the search was conducted from 2014 onwards. Relevant literatures were extracted from PubMed, EMBASE, Cochrane Library databases, and WANFANG DATA (Chinese database) from 2014 to October 2020. The keywords used during the search were: "stomach neoplasm" OR "gastric neoplasms" OR "gastric neoplasm" OR "cancer of stomach" OR "stomach cancers" OR "gastric cancer" OR "gastric cancers" OR "stomach cancer" OR "cancer of the stomach" OR "stomach neoplasms" AND "systemic immune-inflammatory index" OR "SII".

\section{Selection criteria}

The inclusion criteria were as follows: (I) the patients had pathologically confirmed GC. (II) The study divided the enrolled patients into groups according to the SII level and conducted long-term follow-up (>1 year). (III) The patient's outcome was death, recurrence, or status at the end of follow-up; and (IV) randomized controlled tests, case-control studies, or cohort studies using a multivariate proportional hazard model adjusted for survival outcomes. If the data overlapped in more than one study, only the most complete studies were included. Articles were excluded if (I) they were a review, abstract, conference paper, or case report or (II) the data were insufficient for estimating the hazard ratio (HR) and 95\% confidence interval (CI) or (III) HR does not indicate the risk ratio between the different SII groups.

\section{Data extraction}

Article evaluation and data extraction were performed by two independent authors. If a disagreement occurred, a third author was consulted. The following items were recorded: the first author, year of publication, country, the total number of patients, the patients' gender and age, follow-up, cut-off value, TNM stage, and HRs with $95 \%$ CIs.

\section{Quality assessment}

The included studies' qualities were assessed using the Newcastle-Ottawa Scale (NOS) (26) by two independent authors. The NOS consisted of three parts: selection (0-4 points), comparability (0-2 points), and outcome assessment (0-3 points). Studies with NOS scores $\geq 6$ were classified as 


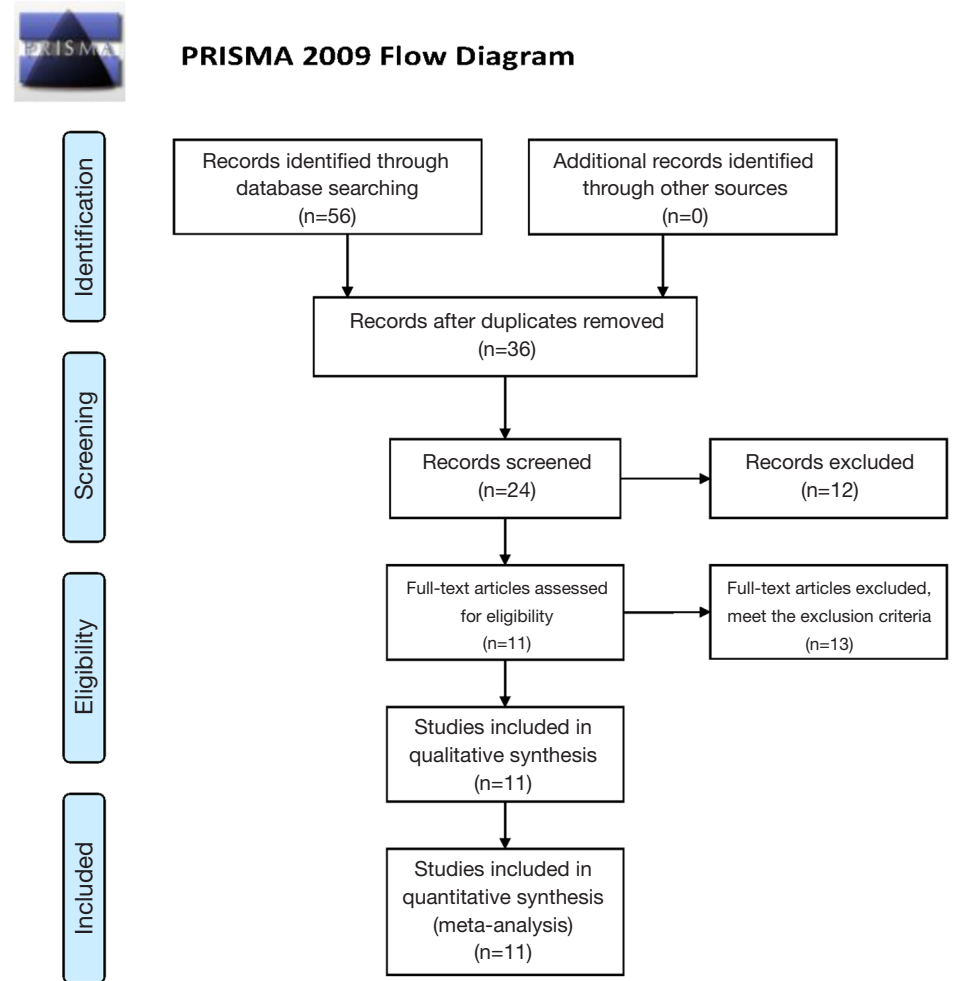

From: Moher D, Liberati A, Tetzlaff J, Altman DG, The PRISMA Group (2009). Preferred Reporting /tems for Systematic Reviews and MetaAnalyses: The PRISMA Statement. PLoS Med 6(7): e1000097, doi:10.1371/journal.pmed1000097

For more information, visit www. prisma-statement.org.

Figure 1 Search, screening, and selection process for studies about prognosis role of SII in GC. SII, systemic immune-inflammatory index; GC, gastric cancer.

high-quality studies.

\section{Statistical analyses}

All data analyses were performed using Stata 12.0 software. The included HRs and 95\% CIs were calculated with the combined effect size. In addition, a pooled HR $>1$ meant that high SII was an unfavorable factor for GC, indicating that a high SII was associated with a poor prognosis. The Cochran's Q test and Higgins' I-squared statistic were performed to assess heterogeneity. P heterogeneity $<0.10$ or $\mathrm{I}^{2}>50 \%$ suggested significant heterogeneity. The randomeffects model was used when significant heterogeneity was observed. Otherwise, the fixed-effects model was used. Subgroup analysis, meta-regression, and sensitivity analysis were used to define heterogeneity. Publication bias was assessed by visual inspection of a Begg's funnel plot. A P value $<0.05$ was considered significant.

\section{Results}

\section{Literature characteristics}

After a preliminary search, 56 articles were found in PubMed, EMBASE, Cochrane Library, and WANFANG DATA. After excluding 20 duplicate articles, 12 irrelevant articles were also excluded after reviewing the abstracts. During full-text analysis, 13 articles met the exclusion criteria and were hence removed. Finally, 11 articles were included in the analysis $(16-24,27,28)$. The study selection process is shown in Figure 1 (29). All 11 selected studies were determined to be of high quality (NOS score range: 6-8 points; Figure 2).

Altogether, 6,925 patients were included, and the articles were published between 2014 and 2020. Seven of 


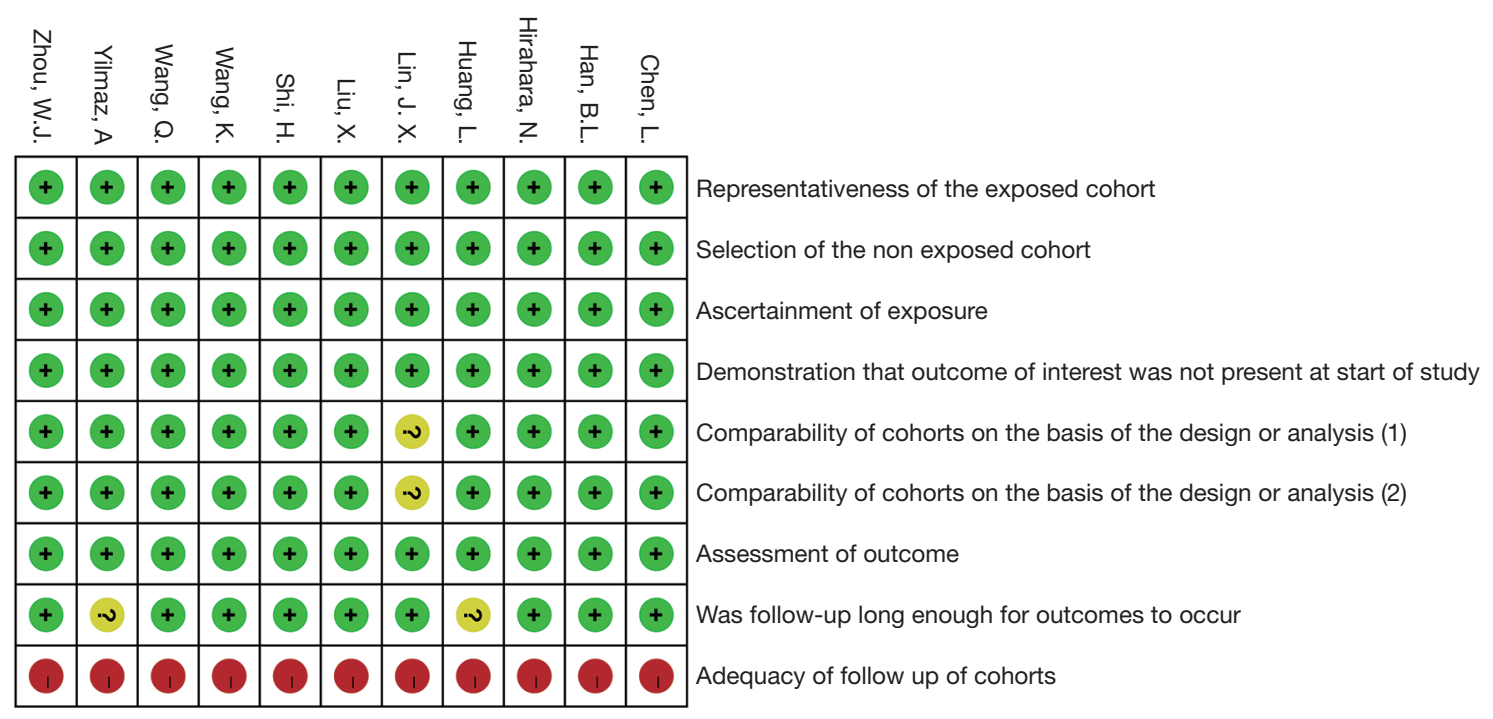

Figure 2 Quality assessment of included studies.

Table 1 Main characteristics of the included studies in this analysis

\begin{tabular}{|c|c|c|c|c|c|c|c|c|c|c|c|}
\hline Author & Year & Country & Size & Gender (M/F) & Age & Follow-up (month) & Cut-off (cells/L) & Stage & Outcome & HR type & NOS \\
\hline Huang $L$ & 2016 & China & 455 & $305 / 150$ & 56 [21-85] & $655[305-1,017]$ day & 572 & $|-||-|||$ & OS & M & 7 \\
\hline Zhou WJ & 2016 & China & 192 & $156 / 36$ & 63 & $47.9[1-100]$ & 543.86 & $|-||-|||$ & os & $\mathrm{U} / \mathrm{M}$ & 7 \\
\hline Chen $\mathrm{L}$ & 2017 & China & 292 & $207 / 85$ & 57 [28-77] & $>60$ & 600 & I-II-III-IV & OS/DFS & $\mathrm{U} / \mathrm{M}$ & 8 \\
\hline Shi H [1] & 2018 & China & 668 & $471 / 217$ & 56 & $36[1-75]$ & 320 & |-||-||| & os & $\mathrm{U} / \mathrm{M}$ & 8 \\
\hline Shi H [2] & 2018 & China & 174 & $131 / 43$ & 56 & 32 [4-69] & 320 & $|-||-|||$ & OS & $\mathrm{U} / \mathrm{M}$ & 8 \\
\hline Han BL & 2019 & China & 1,509 & $1,124 / 385$ & 60 [22-91] & $>60$ & 570.5 & I-II-III-IV & OS & $\mathrm{U} / \mathrm{M}$ & 7 \\
\hline Wang Q & 2019 & China & 182 & $133 / 49$ & $55.7 \pm 9.5$ & $>60$ & 600 & III & OS/DFS & $\mathrm{U} / \mathrm{M}$ & 8 \\
\hline Hirahara N & 2020 & Japan & 212 & $154 / 36$ & 73 [41-90] & $>60$ & 661.9 & $|-||-|||$ & os & U/M & 7 \\
\hline
\end{tabular}

NOS, Newcastle-Ottawa Scale.

them were conducted in China, one in Turkey, and one in Japan. Each study reported the HR and 95\% CI of SII to the OS of patients with GC. Among them, Shi et al. (19) reported two sets of valid data because they divided the selected patients into two groups and calculated their data separately. All selected studies reported the HR of OS, and three articles reported the HR of disease-free survival (DFS). Eight selected studies reported that SII was a risk factor for poor prognosis in patients with GC, whereas three did not. The cut-off value of SII varied in different studies, ranging from $320 \times 10^{9}$ to $802 \times 10^{9}$ cells/L. Information regarding all included articles are listed in Table 1.

\section{Relationship between SII and survival outcomes in GC}

Since the OS results were significantly heterogeneous 


\begin{tabular}{|c|c|c|c|}
\hline Study & & & $\%$ \\
\hline ID & & $\mathrm{HR}(95 \% \mathrm{Cl})$ & Weight \\
\hline Liu, X. (2015) & $a$ & $1.04(0.72,1.48)$ & 8.57 \\
\hline Huang, L. (2016) & $\longrightarrow$ & $1.80(1.17,2.76)$ & 7.55 \\
\hline Zhou, W.J. (2016) & $\longrightarrow$ & $1.58(1.07,2.34)$ & 8.09 \\
\hline Wang, K. (2017) & $\rightarrow$ & $1.55(1.21,1.99)$ & 10.38 \\
\hline Chen, L. (2017) & $\rightarrow$ & $1.64(1.19,2.24)$ & 9.28 \\
\hline Shi, H. (1) (2018) & $\rightarrow$ & $1.61(1.27,2.05)$ & 10.51 \\
\hline Shi, H. (2) (2018) & $\longrightarrow$ & $2.94(1.83,4.73)$ & 6.88 \\
\hline Wang, Q. (2019) & $\stackrel{1}{\rightarrow}$ & $1.88(1.31,2.69)$ & 8.59 \\
\hline Han, B.L. (2019) & $\rightarrow$ & $1.34(1.19,1.51)$ & 12.16 \\
\hline Lin, J.X. (2020) & & $0.84(0.69,1.03)$ & 11.08 \\
\hline Yilmaz, A. (2020) & & $-2.00(0.37,10.86)$ & 1.07 \\
\hline Hirahara, N. (2020) & $\rightarrow$ & $2.19(1.25,3.82)$ & 5.85 \\
\hline Overall (I-squared $=76.5 \%, P=0.000$ ) & $>$ & $1.53(1.27,1.83)$ & 100.00 \\
\hline NOTE: Weights are from random effects analysis & & & \\
\hline 0.0921 & & 10.9 & \\
\hline
\end{tabular}

Figure 3 Forest plot of HR between SII and OS in GC patients. HR, hazard ratio; SII, systemic immune-inflammatory index; OS, overall survival; GC, gastric cancer; CI, confidence interval.

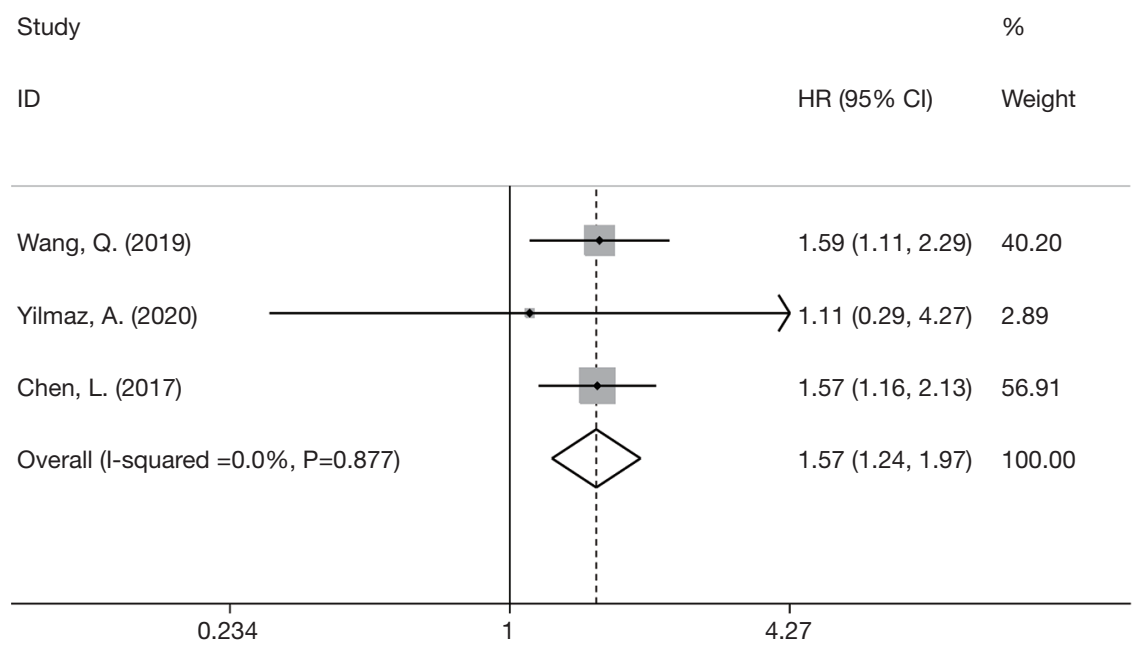

Figure 4 Forest plot of HR between SII and DFS in GC patients. HR, hazard ratio; SII, systemic immune-inflammatory index; DFS, disease-free survival; GC, gastric cancer; CI, confidence interval.

$\left(\mathrm{I}^{2}=76.5 \%, \mathrm{P}_{\mathrm{h}}=0.000\right)$, a random-effects model was chosen in our study for OS. The pooled HR indicated that a higher SII value was significantly associated with worse OS in GC patients (HR: 1.53, 95\% CI: $1.27-1.83$ ), which was consistent with the results of eight of the included studies (Figure 3).
Since the DFS results were not significantly heterogeneous $\left(\mathrm{I}^{2}=0, \mathrm{P}_{\mathrm{h}}=0.877\right)$, a fixed-effects model was chosen in our study for DFS. The pooled HR indicated that a higher SII value was significantly associated with worse DFS in GC patients (HR: 1.57, 95\% CI: 1.24-1.97) (Figure 4). 
Table 2 Subgroup analysis of SII and OS in GC patients

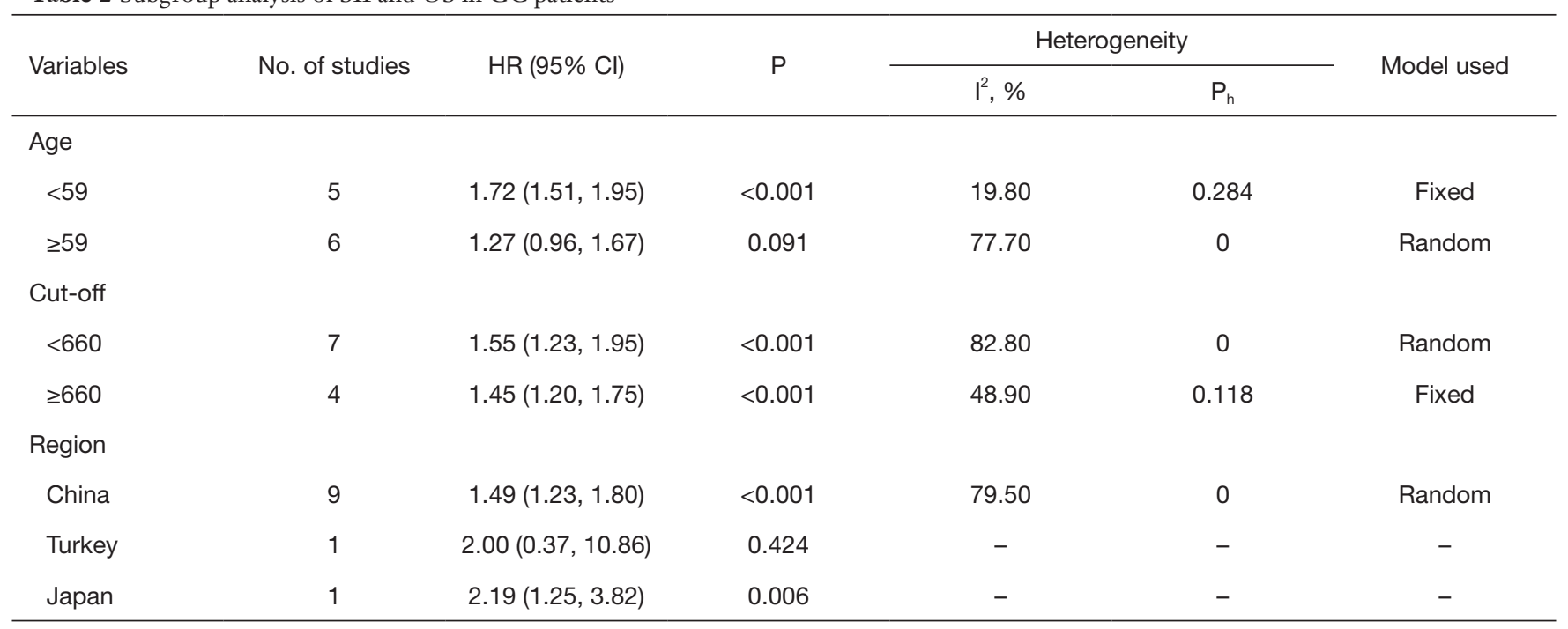

SII, systemic immune-inflammatory index; OS, overall survival; GC, gastric cancer; HR, hazard ratio; Cl, confidence interval.

\section{Subgroup analysis and meta-regression}

In order to explore the source of heterogeneity, a subgroup analysis was conducted based on the median/mean age, cut-off value, and region. The results are shown in Table 2 . Heterogeneity decreased significantly only when grouped by the median/mean age of $<59$ and $\geq 59$ years $\left(\mathrm{I}^{2}=19.80 \%\right.$, $\mathrm{P}_{\mathrm{h}}=0.284$ and $\left.\mathrm{I}^{2}=77.7 \%, \mathrm{P}_{\mathrm{h}}=0\right)$, and the results were quite opposite in these two groups. The HR was 1.72 (95\% CI: $1.51-1.95)$ in patients aged $<59$ years and 1.27 (95\% CI: $0.96-1.67$ ) in patients aged $\geq 59$ years (Figure 5).

Meta-regression analysis indicated that median/mean age was related to heterogeneity $(P>|t|=0.046)$. After introducing the median/mean age, the variance between the studies was $0.042\left(\mathrm{tau}^{2}=0.042\right)$, which can explain the $45.70 \%$ of the source of heterogeneity (adj R-squared $=45.70 \%)$.

\section{Sensitivity analysis and publication bias}

The sensitivity analysis indicated that the results of the combined analysis were robust (Figure 6). The Begg's funnel plots are shown in Figure 7. The P value of Begg's tests was 0.244 , which indicated that there was no publication bias in the current studies.

\section{Discussion}

GC is a highly lethal malignant tumor of the digestive system (1). Although the TNM staging system can predict the prognosis of GC patients, it cannot distinguish patients with the same stage but having different prognoses. Therefore, accurate indices are needed to improve the prognosis of GC patients. The blood routine test-related indices, such as NLR, PLR, and SII are prognostic indicators for tumors, including liver cancer and esophageal cancer, and have been proposed in recent years (4-6). Among them, SII is an index that can better reflect the balance of inflammation and immunity in the human body. Studies have reported that SII is a risk factor for poor prognosis in hepatocellular carcinoma (8), esophageal cancer (9), and small cell lung cancer (30). Moreover, some studies suggest that SII is superior to NLP and PLR $(10,17)$.

Our meta-analysis integrated 11 articles on the relationship of SII and OS of GC patients. Patients with higher SII had worse OS than patients with lower SII (HR: 1.53, 95\% CI: 1.27-1.83). However, in the subgroup analysis for the group $\geq 59$ years, the pooled HR and $95 \%$ $\mathrm{CI}$ indicated that SII might be an ineffective indicator for the OS of GC patients (HR: 1.27, 95\% CI: 0.96-1.67). There are two possible explanations for this observation. First, the number of included studies were limited, and the heterogeneity is high $\left(\mathrm{I}^{2}=77.7 \%, \mathrm{P}_{\mathrm{h}}=0\right)$. Second, the normal SII level in elderly people is lower than that in young people (31); therefore, using the same SII cut-off value may affect results. The cut-off value is an important factor that affects the predictive power of SII. When Huang 


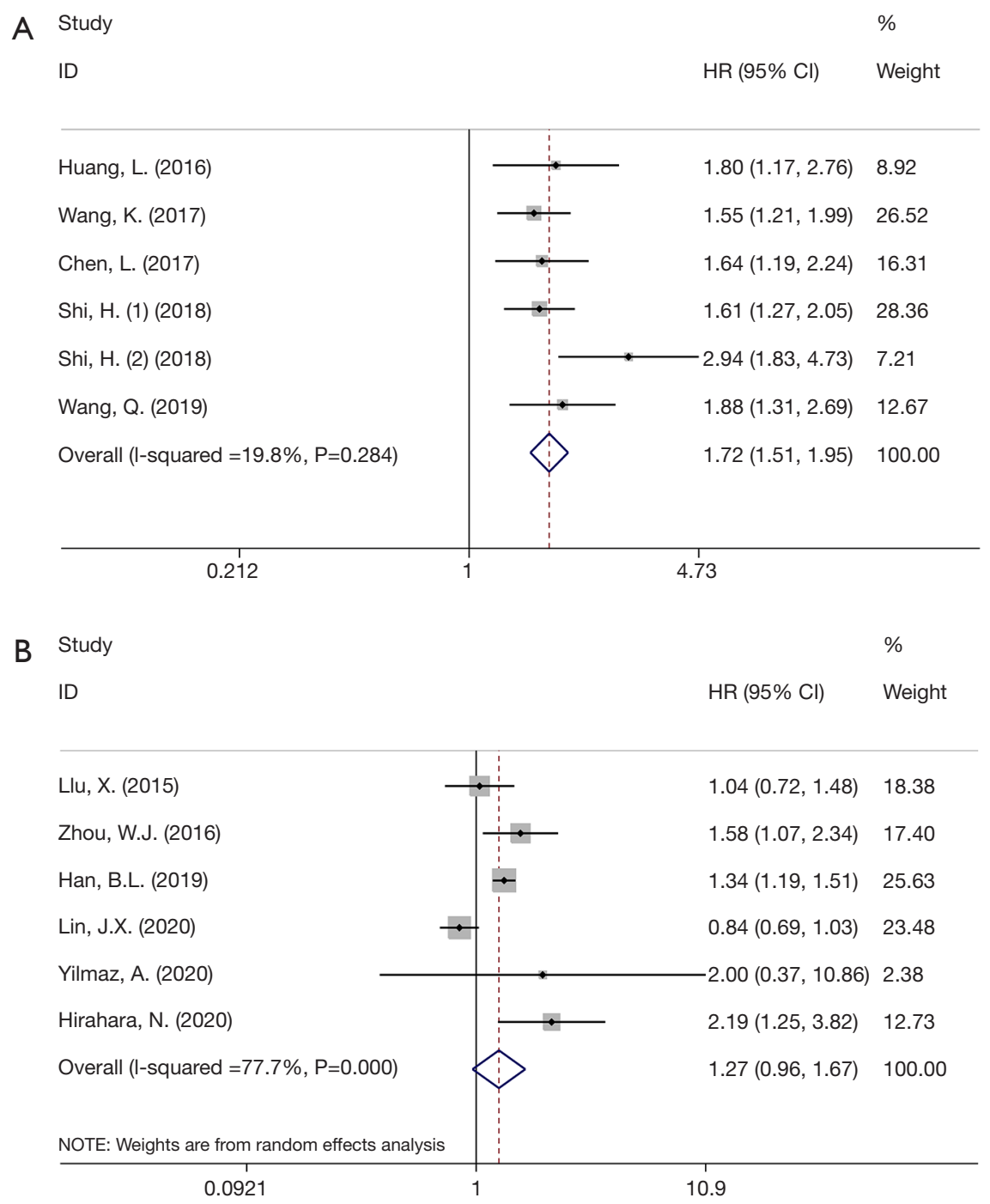

Figure 5 Forest plot of HR between SII and OS stratified by age (<59 vs. 259 ). (A) Subgroup analysis of GC patient age <59; (B) subgroup analysis of GC patient age $\geq 59$. HR, hazard ratio; SII, systemic immune-inflammatory index; OS, overall survival; GC, gastric cancer; CI, confidence interval.

et al. (16) chose $320 \times 10^{9}$ cells $/ \mathrm{L}$ as the cut-off value, they observed that the accuracy in predicting the 3- and 5-year survival rates of GC patients with SII was more accurate than the TNM staging system. While other studies use SII as a continuous variable to explore its significance in cancer patients' OS, their results indicate that SII had no predictive prognostic value $(32,33)$. We observed that the cut-off value suitable for different ages may not be consistent. It is hence necessary to determine the cut-off value suitable for elderly patients to further explore the value of SII in OS prediction. Nevertheless, determining the appropriate cut-off value for different age groups requires further research.

Inflammation is one of the seven characteristics of tumors (34). Inflammatory cells and inflammatory cytokines are important components of the tumor microenvironment. Local inflammation will cause local homeostatic imbalance. Normal tissue cells will thus obtain the possibility of tumor cellularization and even promote the growth and metastasis of existing tumor cells (35). In this regard, inflammatory factors are considered to be an important component of tumor promotion. Unlike inflammation, the monitoring and destruction functions of the body's immune system can 


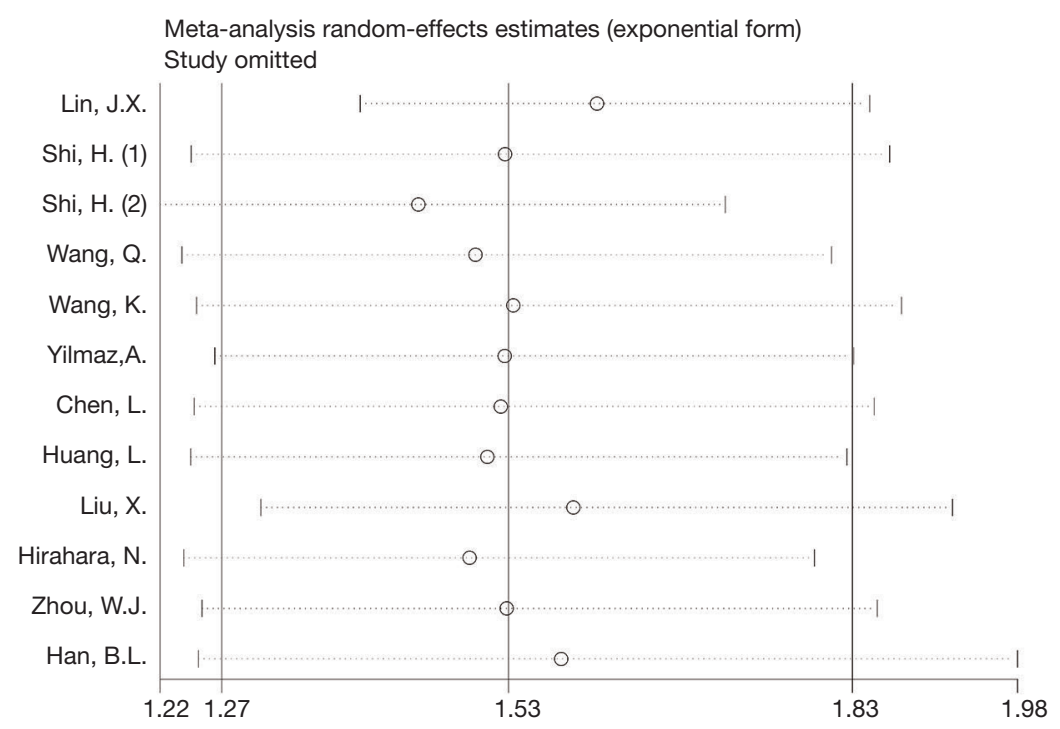

Figure 6 Sensitivity analysis of meta-analysis.

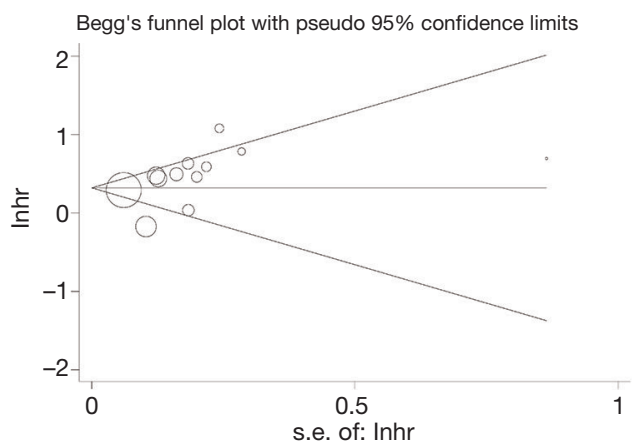

Figure 7 The Begg's funnel plots of meta-analysis.

detect and destroy cells in the body that have a tendency to become tumorigenic (35). Tumor cells can only survive if they escape immunity detection. Although the effects of inflammation and immunity on tumors are not just simply promotion and suppression, the balance of inflammation and immune factors does affect the biological behavior of tumors.

SII is based on the peripheral counts of neutrophils, platelets, and lymphocytes, and it may reflect the balance of inflammation and immune factors in the body. Neutrophils are an important component of the nonspecific immune system and an important inflammatory factor. The cytokines and chemokines they produce can lead to abnormal activation of $\mathrm{T}$ cells and create favorable conditions for tumorigenesis (36-38). Platelets can release adenine and adenylate to protect circulating tumor cells, cause epithelial-mesenchymal transformation, and favor distant metastasis and colonization of tumor cells (39). Lymphocytes are an important component of cellular immunity and can inhibit the occurrence and growth of tumor cells $(40,41)$. Therefore, when SII levels increase, it means that the neutrophil or platelet counts increased, or that the lymphocyte count decreased; i.e., the inflammatory factors in the body are stronger than the immune factors, the survival conditions of the tumor are better, and the risk of poor prognosis is greater. This may be the reason why SII can predict the prognosis of cancer patients.

However, this study has some limitations. First, the pooled HR had significant heterogeneity $\left(\mathrm{I}^{2}=76.5 \%\right.$, $\mathrm{P}_{\mathrm{h}}=0.000$ ). Although age was confirmed to be the source of heterogeneity through subgroup analysis and metaregression, the specific mechanism remains unclear. Second, the SII cut-off values among the included articles were not the same. Although it was not considered the cause of heterogeneity $(\mathrm{P}=0.861)$, it was a potential source of confounding bias. In view of the obvious influence of age on SII, it is hoped that cut-off values suitable for different age groups can be selected, and patients can be divided into subgroups according to age, which can reduce confounding bias caused by different cut-off values. Third, the number of included studies were limited, and most of them were carried out in China, and this could have been a potential source of selection bias. It is hoped that more similar studies 
in different regions and ethnicities can be included in the future to reduce selection bias. We did not find publication bias in our study; however, the possibility of false negatives still remains.

\section{Conclusions}

In summary, the pooled HR indicates that a higher SII in younger patients with GC predicts a poor prognosis. In elderly patients with GC, the significance of SII for prognosis still needs further research.

\section{Acknowledgments}

Thanks to Editage by CACTUS for its help in editing the article

Funding: None.

\section{Footnote}

Reporting Checklist: The authors have completed the PRISMA 2009 reporting checklist. Available at http:// dx.doi.org/10.21037/jgo-20-252

Conflicts of Interest: All authors have completed the ICMJE uniform disclosure form (available at http://dx.doi. org/10.21037/jgo-20-252). The authors have no conflicts of interest to declare.

Ethical Statement: The authors are accountable for all aspects of the work in ensuring that questions related to the accuracy or integrity of any part of the work are appropriately investigated and resolved.

Open Access Statement: This is an Open Access article distributed in accordance with the Creative Commons Attribution-NonCommercial-NoDerivs 4.0 International License (CC BY-NC-ND 4.0), which permits the noncommercial replication and distribution of the article with the strict proviso that no changes or edits are made and the original work is properly cited (including links to both the formal publication through the relevant DOI and the license). See: https://creativecommons.org/licenses/by-nc-nd/4.0/.

\section{References}

1. Bray F, Ferlay J, Soerjomataram I, et al. Global cancer statistics 2018: GLOBOCAN estimates of incidence and mortality worldwide for 36 cancers in 185 countries. CA Cancer J Clin 2018;68:394-424.

2. Paoletti X, Oba K, Burzykowski T, et al. Benefit of adjuvant chemotherapy for resectable gastric cancer: a meta-analysis. JAMA 2010;303:1729-37.

3. Digklia A, Wagner AD. Advanced gastric cancer: current treatment landscape and future perspectives. World J Gastroenterol 2016;22:2403-14.

4. Chen L, Hao Y, Zhu L, et al. Monocyte to lymphocyte ratio predicts survival in patients with advanced gastric cancer undergoing neoadjuvant chemotherapy. Onco Targets Ther 2017;10:4007-16.

5. Feng JF, Huang Y, Chen QX. Preoperative platelet lymphocyte ratio (PLR) is superior to neutrophil lymphocyte ratio (NLR) as a predictive factor in patients with esophageal squamous cell carcinoma. World J Surg Oncol 2014;12:58.

6. Ock CY, Nam AR, Lee J, et al. Prognostic implication of antitumor immunity measured by the neutrophillymphocyte ratio and serum cytokines and angiogenic factors in gastric cancer. Gastric Cancer 2017;20:254-62.

7. Shao Y, Geng Y, Gu W, et al. Assessment of lymph node ratio to replace the $\mathrm{pN}$ categories system of classification of the TNM system in esophageal squamous cell carcinoma. J Thorac Oncol 2016;11:1774-84.

8. Wang B, Huang Y, Lin T. Prognostic impact of elevated pre-treatment systemic immune-inflammation index (SII) in hepatocellular carcinoma: a meta-analysis. Medicine (Baltimore) 2020;99:e18571.

9. Zhang Y, Xiao G, Wang R. Clinical significance of systemic immune-inflammation index (SII) and C-reactive proteinto-albumin ratio (CAR) in patients with esophageal cancer: a meta-analysis. Cancer Manag Res 2019;11:4185-200.

10. Chen JH, Zhai ET, Yuan YJ, et al. Systemic immuneinflammation index for predicting prognosis of colorectal cancer. World J Gastroenterol 2017;23:6261-72.

11. Ji Y, Wang H. Prognostic prediction of systemic immuneinflammation index for patients with gynecological and breast cancers: a meta-analysis. World J Surg Oncol 2020;18:197.

12. Huang $Y$, Gao $Y, W u ~ Y$, et al. Prognostic value of systemic immune-inflammation index in patients with urologic cancers: a meta-analysis. Cancer Cell Int 2020;20:499.

13. Yan Q, Ertao Z, Zhimei Z, et al. Systemic immuneinflammation index (SII): a more promising inflammationbased prognostic marker for patients with synchronic colorectal peritoneal carcinomatosis. J Cancer 2020;11:5264-72. 
14. Yang R, Chang Q, Meng X, et al. Prognostic value of systemic immune-inflammation index in cancer: a metaanalysis. J Cancer 2018;9:3295-302.

15. Fest J, Ruiter R, Mulder M, et al. The systemic immuneinflammation index is associated with an increased risk of incident cancer-a population-based cohort study. Int J Cancer 2020;146:692-8.

16. Huang L, Liu S, Lei Y, et al. Systemic immuneinflammation index, thymidine phosphorylase and survival of localized gastric cancer patients after curative resection. Oncotarget 2016;7:44185-93.

17. Wang K, Diao F, Ye Z, et al. Prognostic value of systemic immune-inflammation index in patients with gastric cancer. Chin J Cancer 2017;36:75.

18. Chen L, Yan Y, Zhu L, et al. Systemic immuneinflammation index as a useful prognostic indicator predicts survival in patients with advanced gastric cancer treated with neoadjuvant chemotherapy. Cancer Manag Res 2017;9:849-67.

19. Shi H, Jiang Y, Cao H, et al. Nomogram based on systemic immune-inflammation index to predict overall survival in gastric cancer patients. Dis Markers 2018;2018:1787424.

20. Wang Q, Zhu D. The prognostic value of systemic immune-inflammation index (SII) in patients after radical operation for carcinoma of stomach in gastric cancer. J Gastrointest Oncol 2019;10:965-78.

21. Hirahara N, Tajima Y, Matsubara T, et al. Systemic immune-inflammation index predicts overall survival in patients with gastric cancer: a propensity score-matched analysis. J Gastrointest Surg 2020. [Epub ahead of print].

22. Lin JX, Wang ZK, Huang YQ, et al. Dynamic changes in pre- and postoperative levels of inflammatory markers and their effects on the prognosis of patients with gastric cancer. J Gastrointest Surg 2021;25:387-96.

23. Y1lmaz A, Mirili C, Tekin SB, et al. The ratio of hemoglobin to red cell distribution width predicts survival in patients with gastric cancer treated by neoadjuvant FLOT: a retrospective study. Ir J Med Sci 2020;189:91-102.

24. Liu X, Sun X, Liu J, et al. Preoperative C-reactive protein/ albumin ratio predicts prognosis of patients after curative resection for gastric cancer. Transl Oncol 2015;8:339-45.

25. Liberati A, Altman DG, Tetzlaff J, et al. The PRISMA statement for reporting systematic reviews and metaanalyses of studies that evaluate healthcare interventions: explanation and elaboration. BMJ 2009;339:b2700.

26. Stang A. Critical evaluation of the Newcastle-Ottawa scale for the assessment of the quality of nonrandomized studies in meta-analyses. Eur J Epidemiol 2010;25:603-5.

27. Zhou WJ, Wu J, Wang Q, et al. Evaluate the correlation between preoperative systemic immune-inflammation index and prognosis of patients after curative resection for cardia gastric cancer. Chin Med Biotechnol 2016;11:510-4.

28. Han BL, Wang Y, Xue Y. Effect of preoperative systemic immune inflammation index on the prognosis of postoperative gastric cancer patients. Chinese Journal of General Surgery 2019;34:306-9.

29. Moher D, Liberati A, Tetzlaff J, et al. Preferred reporting items for systematic reviews and meta-analyses: the PRISMA statement. PLoS Med 2009;6:e1000097.

30. Hong X, Cui B, Wang M, et al. Systemic immuneinflammation index, based on platelet counts and neutrophil-lymphocyte ratio, is useful for predicting prognosis in small cell lung cancer. Tohoku J Exp Med 2015;236:297-304.

31. Luo H, He L, Zhang G, et al. Normal reference intervals of neutrophil-to-lymphocyte ratio, platelet-to-lymphocyte ratio, lymphocyte-to-monocyte ratio, and systemic immune inflammation index in healthy adults: a large multi-center study from Western China. Clin Lab 2019. doi: 10.7754/Clin.Lab.2018.180715.

32. Li Z, Li S, Ying X, et al. The clinical value and usage of inflammatory and nutritional markers in survival prediction for gastric cancer patients with neoadjuvant chemotherapy and D2 lymphadenectomy. Gastric Cancer 2020;23:540-9.

33. Guner A, Kim SY, Yu JE, et al. Parameters for predicting surgical outcomes for gastric cancer patients: simple is better than complex. Ann Surg Oncol 2018;25:3239-47.

34. Grivennikov SI, Greten FR, Karin M. Immunity, inflammation, and cancer. Cell 2010;140:883-99.

35. Elinav E, Nowarski R, Thaiss CA, et al. Inflammationinduced cancer: crosstalk between tumours, immune cells and microorganisms. Nat Rev Cancer 2013;13:759-71.

36. Tan KW, Chong SZ, Wong FH, et al. Neutrophils contribute to inflammatory lymphangiogenesis by increasing VEGF-A bioavailability and secreting VEGF-D. Blood 2013;122:3666-77.

37. Najmeh S, Cools-Lartigue J, Rayes RF, et al. Neutrophil extracellular traps sequester circulating tumor cells via beta1-integrin mediated interactions. Int J Cancer 2017;140:2321-30.

38. Müller I, Munder M, Kropf P, et al. Polymorphonuclear neutrophils and T lymphocytes: strange bedfellows or brothers in arms? Trends Immunol 2009;30:522-30.

39. Labelle M, Begum S, Hynes RO. Platelets guide the 
formation of early metastatic niches. Proc Natl Acad Sci U S A 2014;111:E3053-61.

40. Ogiya R, Niikura N, Kumaki N, et al. Comparison of tumor-infiltrating lymphocytes between primary and

Cite this article as: Fu S, Yan J, Tan Y, Liu D. Prognostic value of systemic immune-inflammatory index in survival outcome in gastric cancer: a meta-analysis. J Gastrointest Oncol 2021;12(2):344-354. doi: 10.21037/jgo-20-252 metastatic tumors in breast cancer patients. Cancer Sci 2016;107:1730-5.

41. Ferrone C, Dranoff G. Dual roles for immunity in gastrointestinal cancers. J Clin Oncol 2010;28:4045-51. 